\title{
Género en los lineamientos institucionales de atención a las jóvenes en conflicto con la ley. Aspectos para políticapública ${ }^{1}$
}

\author{
Gender in the Institutional Guidelines of Attention to Young Women in Conflict \\ with the Law. AspectsforPublicPolicy
}

\author{
Luz Angela Cortina Roa \\ Universidad del Rosario, Bogotá, Colombia. \\ luz.cortina@urosario.edu.co |https://orcid.org/0000-0002-2977-1250
}

\begin{abstract}
Alba Nubia Rodríguez Pizarro
Universidad del Valle, Cali, Colombia.

alba.rodriguez@correounivalle.edu.co / https://orcid.org/0000-0003-1055-9636
\end{abstract}

Recibido: 16 de junio de 2020. Aprobado: 22 de septiembre de 2020.

DOI: $10.25100 /$ lamanzanadeladiscordia.v15i2.9879

Artículo de investigación

¿Cómo citar este artículo? / How to quote this article?

Cortina, Luz Angela, y Rodríguez, Alba Nubia. (2020). Género en los lineamientos

institucionales de atención a las jóvenes en conflicto con la ley. Aspectos para políticapública. $L a$ Manzana de la Discordia, 15(2),1-29. 10.25100/lamanzanadeladiscordia.v15i2.9879

\footnotetext{
${ }^{1}$ Artículo de investigación resultado del proyecto de investigación de tesis doctoral "La configuración de las huellas de intervención intrainstitucionales en género y ocupación en mujeres jóvenes privadas de la libertad en el Eje cafetero y suroccidente colombiano en el año 2018", financiado por Colciencias y la Vicerrectoria de Investigaciones de la Universidad del Valle. El proyecto fue ejecutado durante el período 2016-2018 y dirigido por Alba Nubia Rodríguez Pizarro.
} 


\title{
Resumen
}

El propósito de este artículo es presentar el análisis con perspectiva de género de los lineamientos institucionales vigentes que rigen la atención de las jóvenes en conflicto con la ley privadas de la libertad; en la misma línea, analizar la materialización de los principios de dichos lineamientos en los Centros de Atención Especializados y, finalmente, plantear algunos aspectos para política pública respecto a la importancia de incluir en los lineamientos institucionales para la atención y la intervención no solo el género como concepto, sino como perspectiva.

El propósito de este artículo es presentar el análisis con perspectiva de género de los lineamientos institucionales vigentes que rigen la atención de las jóvenes en conflicto con la ley privadas de la libertad;en la misma línea, analizar la materialización de los principios de dichos lineamientos en los Centros de Atención Especializados. El método, a partir de la perspectiva cualitativa, fue el análisis documental del acervo legal que respalda las acciones intrainstitucionales, en este se halla la generalización, inclusive en el lenguaje, de lo masculino sobre lo femenino, así como de las acciones a desarrollar con los hombres jóvenes sobre los necesarias a desarrollar con las mujeres; es decir se invisibilizan sus necesidades y particularidades, siendo solo diferencial aquellas que refuerzan el papel de la mujer como cuidadora y reproductora de la especie. Derivado de lo anterior, se plantean algunos aspectos para política pública respecto a la importancia de incluir en los lineamientos institucionales para la atención y la intervención no solo el género como concepto, sino como perspectiva.

Palabras clave: género; mujeres jóvenes en conflicto con la ley; lineamientos de atención.

\begin{abstract}
The purpose of this article is to present the analysis from a gender perspective of the current institutional guidelines that govern the care of young women in conflict with the law deprived of liberty; Along the same lines, analyze the materialization of the principles of said guidelines in Specialized Care Centers. The method, from the qualitative perspective, was the documentary analysis of the legal heritage that supports the intra-institutional actions, in this is the generalization, including in language, of the masculine over the feminine, as well as the actions to be developed with young men on those necessary to develop with women; In other words, their needs and particularities are made invisible, being only differential those that reinforce the role


of women as caretakers and reproducers of the species. Derived from the above, some aspects are raised for public policy regarding the importance of including gender in the institutional guidelines for care and intervention not only as a concept, but also as a perspective.

Keywords: gender;young women in conflict with the law;care guidelines.

\section{Introducción}

En 2018, según cifras de la Organización de Naciones Unidas, las mujeres conforman el 49,54\% de la población mundial, siendo aproximadamente 3.761 millones. En Colombia, según el DANE (2018), para el mismo año, el 51,2\% de la población del país son mujeres, de ellas, el 8,5\% se encuentran entre los 15 y los 24 años. Según cifras emitidas por el Instituto Colombiano de Bienestar Familiar (2018), en el periodo comprendido entre enero y junio de 2018, el 0,06\% de las mujeres entre los 15 y los 19 años cometieron algún delito. Así mismo, de la población joven infractora, las mujeres representan el 14\%, es decir, 1.197 mujeres, frente al $86 \%$ de los varones que corresponde a 7.960 hombres menores de edad. Esto evidencia una diferencia sustancial de 73 puntos porcentuales entre estos dos géneros.

Las mujeres jóvenes que han realizado acciones en contra de la ley en Colombia reciben diferentes sanciones de acuerdo con la falta cometida y al concepto del juez que valora dicha falta, que, de acuerdo con el Instituto Colombiano de Bienestar Familiar (2018), las de mayor preponderancia en el territorio colombiano son el hurto, el tráfico, la fabricación o porte de estupefacientes y la violencia intrafamiliar. Para atender e intervenir esta situación, el Estado creó el Sistema de Responsabilidad Penal Adolescente (SRPA), definido como:

El conjunto de principios, normas, procedimientos, autoridades judiciales y especializadas y entes administrativos que rigen o intervienen en la investigación y juzgamiento de delitos cometidos por personas que tengan entre catorce (14) y dieciocho (18) años al momento de cometer el hecho punible; cuya finalidad es que tanto el proceso como las medidas que se tomen sean de carácter pedagógico, específico y diferenciado respecto del sistema de adultos, conforme a la protección integral. El proceso deberá garantizar la justicia restaurativa, la verdad y la reparación del daño (Congreso de Colombia, 2006. Art.139). 
El Sistema de Responsabilidad Penal Adolescente (SRPA)rige en el ámbito nacional y su aplicación la realizan los jueces de garantías y de conocimiento, quienes se encargan de determinar la sanción a las jóvenes de acuerdo con el delito cometido. Entre las medidas sancionatorias, se encuentra la privación de la libertad en los Centros de Atención Especializados (CAE), que pueden definirse como "instituciones totales" (Goffman, 2001, p.13), como un “conjunto de aparatos y procedimientos de negociaciones orientadas a la producción de reglas y decisiones legítimas" (Dubet, 2006, p. 31), y como un "agrupamiento configurado por reglamentos establecidos racionalmente" (Weber, 2014, p. 55).

En el marco del Sistema de Responsabilidad Penal Adolescente (SRPA) se dan las medidas sancionatorias de acuerdo con la magnitud del delito. Al tener presente esto, se configura en el final de una cadena circular que inicia desde la misma sociedad, que hace el llamado al Estado para que intervenga las situaciones de infracción a la ley por parte de hombres y mujeres jóvenes. El Estado, después de desarrollar procesos internos, revisión de políticas y cumplir con los convenios internacionales, concibe lineamientos sancionatorios y de resocialización que, dependiendo de la intencionalidad final, pueden o no lograr responder al llamado de la sociedad.

Tanto determinar como ejecutar los lineamientos ${ }^{2}$, tiene innumerables aristas para la aplicación y el análisis, pues depende del gobierno e implementadores, así como de las particularidades de los operadores de turno que hacen la ejecución compleja y casi que impredecible.Es así como, pese a fundamentarse en lineamientos institucionales cuya supervisión está a cargo del Instituto Colombiano de Bienestar Familiar (ICBF), los operadores - ya sean laicos o religiosos - imprimen su particularidad.

Los operadores mencionados son aquellas Organizaciones No Gubernamentales (ONG)pertenecientes al Tercer sectorcontratadaspor el Estado, para desarrollar lo que plantea Foucault (2003) como la ejecución de la pena.Es decir, el Estado se descarga de la puesta en marcha del castigo y resocialización, delegándoloen dichasorganizaciones contratadas para la ejecución de esoslineamientos, que a su vez están influenciadas por políticas internas

\footnotetext{
${ }^{2}$ Los lineamientos y leyes a los que se hace referencia sonlos que hasta el momento reglamentan la intervención con las jóvenes privadas de la libertad en el Sistema de Responsabilidad Penal Adolescente: Ley 1098 de 2006 ; Ley 1577 de 2012; Decreto 860 de 2010; Decreto 2383 de 2015; Lineamiento Modelo de Atención para Adolescentes y Jóvenes en Conflicto con la Ley-Srpa; Lineamiento de Servicios para Medidas y Sanciones del Proceso Judicial Srpa.
} 
institucionales, intereses particulares, de carácter religioso o laico y en ocasiones intereses políticos,que permean la implementación de los lineamientos, así como la relación que las entidades ejecutoras establecen con el Estado.

Con relación a lo anterior, este escrito pretende responder a la pregunta sobre la inclusión de la perspectiva de género en los lineamientosconcebidos por el Estado que reglamentan el proceso de intervención con las jóvenes privadas de la libertad en los Centros de Atención Especializados.

Como parte de la investigación desarrollada para la tesis doctoral titulada "La configuración de las huellas de intervención intrainstitucionales en género y ocupación en mujeres jóvenes privadas de la libertad en el Eje cafetero y suroccidente colombiano en el año $2018^{\text {”3 }}$, se llevó a cabo el análisis documental de las leyes y lineamientos ideados y vigilados por el Estado (y el Instituto Colombiano de Bienestar Familiar en representación de este), vigentes que delimitan y que hasta el momento reglamentan la intervención con las jóvenes entre 14 y 18 años privadas de la libertad.Estos son:

〜 Ley 1098 de 2006.

〜 Ley 1577 de 2012.

Decreto 860 de 2010.

Decreto 2383 de 2015.

Lineamiento Modelo de Atención para Adolescentes y Jóvenes en Conflicto con la Ley-Srpa. Lineamiento de Servicios para Medidas y Sanciones del Proceso Judicial Srpa.

El análisis documental se realizó con metodología cualitativa, de diseño transeccional y la investigación realizada con dos niveles de análisis; el primero documental y el segundocon técnicas participativas.

Acorde con el estudio realizado, el propósito de este artículo es analizar los lineamientos institucionales vigentes que rigen la atención a las jóvenes privadas de la libertadpor hallarse en conflicto con la ley. En virtud de lo anterior,se pretende plantear algunos aspectos relevantes para la construcción de políticas públicasrelacionados con la importancia de incluiren estas, no solo el

\footnotetext{
${ }^{3}$ Investigación que fue financiada por Colciencias y por la Vicerrectoría de Investigaciones de la Universidad del Valle en el periodo 2015-2019 y dirigida por Alba Nubia Rodríguez Pizarro.
} 
género como concepto, sino como perspectivade atención e intervención con las mujeres jóvenes privadas de la libertad en el SRPA.

Para el desarrollo del propósito trazado se retomanplanteamientos de Foucault (2003), entre estos aquel que enuncia al Estado como garantede bienestar;no obstante, para el caso colombiano dicho Estado difícilmente garantiza el bienestar de sus ciudadanos. De lo contrario, sus políticas capitalistas con fines de privatización, desregularización y liberalización tienden a estar en continuo detrimento del bienestar de cada uno de los miembros de la sociedad, en cuyo nivel más inferior se encuentran las mujeres (De Sousa, 2006). A lo anterior se suma el desconocimiento de la corresponsabilidad del Estado, la sociedad y la familia en este asunto; de forma tal, que se diluye el bienestar de las jóvenes quedando entonces delegado a ellas mismas.

Es así como se pretende, al analizar la reglamentación vigente, rastrear asuntos como ¿qué encontramos en las leyes, resoluciones, decretos y documentos del CONPES? ¿qué dice la voz de la institucionalidad? ¿están presentes las particularidades de las mujeres? ¿cómo se entiende y qué lugar se otorga a las mujeres jóvenes?

\section{Análisis de las leyes vigentes que reglamentan el Sistema de Responsabilidad Penal Adolescente colombiano entre el 2006 y el 2018}

La intervención con las jóvenes en conflicto con la ley es reglamentada por leyes, normas y documentos CONPES establecidos por instituciones estatales como el Congreso de la República, el Departamento de Planeación Nacional y el Instituto Colombiano de Bienestar Familiar; encargadas estas de normatizar y ofrecer los lineamientos de atención a los jóvenes ${ }^{4}$ incluidos en el Sistema de Responsabilidad Penal Adolescente.

Las veintidós normas revisadas fueron escogidas por tener relación directa de primer orden con el Sistema de Responsabilidad Penal Adolescente, específicamente con la atención los jóvenes incluidos en este. El listado y descripción general de cada una de ellas se presenta en la tabla 1.

\footnotetext{
${ }^{4}$ Las normativas mencionadas generalizan en "los adolescentes y los jóvenes" a las adolescentes y las jóvenes incluidas en el Sistema de Responsabilidad Penal Adolescente. 
Género en los lineamientos institucionales de atención a las jóvenes en conflicto con la ley. Aspectos para políticapública

Tabla 1. Normas del Sistema de Responsabilidad Penal Adolescente

\begin{tabular}{|c|c|c|}
\hline Norma & $\begin{array}{l}\text { Fecha de } \\
\text { expedición }\end{array}$ & Tema que regula \\
\hline Ley 1098 de 2006 & $08 / 11 / 2006$ & Por la cual se expide el Código de la Infancia y la Adolescencia \\
\hline Ley 1453 de 2011 & $24 / 06 / 2011$ & $\begin{array}{l}\text { Por medio de la cual se reforma el Código Penal, el Código de Procedimiento Penal, el } \\
\text { Código de Infancia y Adolescencia, las reglas sobre extinción de dominio y se dictan } \\
\text { otras disposiciones en materia de seguridad. }\end{array}$ \\
\hline Ley 1577 de 2012 & $20 / 09 / 2012$ & $\begin{array}{l}\text { Por la cual se establecen estímulos tributarios y otros, con el fin de adoptar medidas } \\
\text { especiales para la rehabilitación e inclusión social de jóvenes de alto grado de } \\
\text { emergencia social, pandillismo y violencia juvenil. }\end{array}$ \\
\hline $\begin{array}{l}\text { Decreto } 860 \text { de } \\
2010\end{array}$ & $18 / 03 / 2010$ & $\begin{array}{l}\text { Por el cual se reglamenta parcialmente la Ley } 1098 \text { de } 2006 \text { en cuanto a las obligaciones } \\
\text { del Estado, la sociedad y la Familia en la prevención de la comisión de infracciones a la } \\
\text { ley penal por parte de niños, niñas y adolescentes y su reincidencia, así como las } \\
\text { responsabilidades de los padres o personas responsables del cuidado de los menores de } \\
\text { edad que han cometido tales infracciones, dentro de los procesos administrativos o } \\
\text { penales que se adelanten por las autoridades. }\end{array}$ \\
\hline $\begin{array}{l}\text { CONPES No. } \\
3629 \text { de } 2009\end{array}$ & $14 / 12 / 2009$ & $\begin{array}{l}\text { Sistemas de Responsabilidad Penal para Adolescentes -SRPA: Política de Atención al } \\
\text { Adolescente en Conflicto con la ley. }\end{array}$ \\
\hline $\begin{array}{l}\text { Resolución No. } \\
1301 \text { de } 2010\end{array}$ & $19 / 03 / 2010$ & $\begin{array}{l}\text { Por la cual se aprueba el Lineamiento Técnico Administrativo para la atención de los } \\
\text { adolescentes en el Sistema de Responsabilidad Penal para Adolescentes. }\end{array}$ \\
\hline $\begin{array}{l}\text { Resolución No. } \\
3741 \text { de } 2011\end{array}$ & $31 / 08 / 2011$ & $\begin{array}{l}\text { Por la cual se modifica el Lineamiento Técnico Administrativo para la atención de los } \\
\text { adolescentes en el Sistema de Responsabilidad Penal para Adolescentes. }\end{array}$ \\
\hline $\begin{array}{l}\text { Resolución No. } \\
3454 \text { de } 2011\end{array}$ & $08 / 08 / 2011$ & $\begin{array}{l}\text { Por la cual se establece el Marco Pedagógico para los Servicios de Atención del Sistema } \\
\text { de Responsabilidad Penal para Adolescentes }\end{array}$ \\
\hline Ley 1760 & $06 / 07 / 2015$ & $\begin{array}{l}\text { Por medio de la cual se modifica parcialmente la ley } 906 \text { de } 2004 \text { en relación con las } \\
\text { medidas de aseguramiento privativas de la libertad }\end{array}$ \\
\hline Decreto No.1885 & $21 / 09 / 2015$ & $\begin{array}{l}\text { Por el cual se crea el Sistema Nacional de Coordinación de Responsabilidad Penal para } \\
\text { Adolescentes (Sncrpa) y se dictan otras disposiciones }\end{array}$ \\
\hline Decreto No.2383 & $11 / 12 / 2015$ & $\begin{array}{l}\text { Por el cual se reglamenta la prestación del servicio educativo en el marco del Sistema de } \\
\text { Responsabilidad Penal para Adolescentes y se adiciona al Decreto } 1075 \text { de } 2015 \text { - Único } \\
\text { Reglamentario del Sector Educación }\end{array}$ \\
\hline $\begin{array}{l}\text { Resolución No. } \\
1522\end{array}$ & $23 / 02 / 2016$ & $\begin{array}{l}\text { Por la cual se aprueba el Lineamiento Técnico Modelo de Atención para Adolescentes y } \\
\text { Jóvenes en Conflicto con la Ley - SRPA. }\end{array}$ \\
\hline $\begin{array}{l}\text { Resolución No. } \\
1512\end{array}$ & $23 / 02 / 2016$ & $\begin{array}{l}\text { Por la cual se aprueba el Lineamiento Técnico de Servicios para Medidas y Sanciones } \\
\text { del Proceso Judicial SRPA. }\end{array}$ \\
\hline $\begin{array}{l}\text { Resolución No. } \\
1521\end{array}$ & $23 / 02 / 2016$ & $\begin{array}{l}\text { Por la cual se aprueba el Lineamiento Técnico de Servicios para Medidas y Sanciones } \\
\text { del Proceso Judicial SRPA }\end{array}$ \\
\hline $\begin{array}{l}\text { Resolución No. } \\
5668\end{array}$ & $15 / 06 / 2016$ & $\begin{array}{l}\text { Por la cual se modifica el Lineamiento Técnico Modelo de Atención para Adolescentes y } \\
\text { Jóvenes en Conflicto con la Ley - SRPA aprobado mediante Resolución } 1522 \text { del } 23 \text { de }\end{array}$ \\
\hline
\end{tabular}




\begin{tabular}{|c|c|c|}
\hline & & febrero de 2016 \\
\hline $\begin{array}{l}\text { Resolución No. } \\
5666\end{array}$ & $15 / 06 / 2016$ & $\begin{array}{l}\text { Por la cual se modifica el Lineamiento Técnico de Medidas Complementarias y/o de } \\
\text { Restablecimiento en Administración de Justicia aprobado mediante Resolución } 1512 \text { del } \\
23 \text { de febrero de } 2016 .\end{array}$ \\
\hline $\begin{array}{l}\text { Resolución No. } \\
5667\end{array}$ & $15 / 06 / 2016$ & $\begin{array}{l}\text { Por la cual se modifica el Lineamiento Técnico de Servicios para Medidas y Sanciones } \\
\text { del Proceso Judicial SRPA aprobado mediante Resolución } 1521 \text { del } 23 \text { de febrero de } \\
2016 .\end{array}$ \\
\hline \multicolumn{3}{|c|}{ Lineamiento de servicios para medidas y sanciones del proceso judicial Srpa } \\
\hline $\begin{array}{l}\text { Resolución No. } \\
0328\end{array}$ & $26 / 01 / 2017$ & $\begin{array}{l}\text { Por la cual se aprueba la modificación del Lineamiento Técnico Modelo de Atención } \\
\text { para } \\
\text { Adolescentes y Jóvenes en Conflicto con la Ley (SRPA) aprobado mediante Resolución } \\
5668 \text { del } 15 \text { de junio de } 2016 \text {. }\end{array}$ \\
\hline \multicolumn{3}{|c|}{ Lineamiento modelo de atención para adolescentes y jóvenes en conflicto con la Ley-Srpa } \\
\hline $\begin{array}{lll}\text { Ley } & 1878 & \text { De } \\
2018 & \end{array}$ & $09 / 01 / 2018$ & $\begin{array}{l}\text { Por medio de la cual se modifican algunos artículos de la Ley } 1098 \text { de 2006, por la cual } \\
\text { se expide el Código de la Infancia y la Adolescencia, y se dictan otras disposiciones. }\end{array}$ \\
\hline
\end{tabular}

Fuente: elaboración propia.

Al realizar el análisis, se encontró que dieciséis $(72,72 \%)$ de las normas escogidas y analizadas no hacían referencia específica a los jóvenes en el Sistema de Responsabilidad Penal Adolescente; algunas se centraban en aspectos normativos o presupuestales y en la creación del Sistema Nacional de Coordinación de Responsabilidad Penal para Adolescentes - SNCRPA-; y otras, eran los actos administrativos por los cuales se aprobaban, derogaban o modificaban los lineamientos de atención existentes.

De acuerdo con lo anterior, las leyes y lineamientos que se consideraron adecuados para los fines del presente análisis fueron:

〜 Ley 1098 de 2006.

～Ley 1577 de 2012.

～Decreto 860 de 2010.

～Decreto 2383 de 2015.

Lineamiento Modelo de Atención para Adolescentes y Jóvenes en Conflicto con la Ley-Srpa.

Lineamiento de Servicios para Medidas y Sanciones del Proceso Judicial Srpa 
El análisis de las leyes, las normas y los decretos mencionados se realizó a partir del género como categoría central,que si bien ha sido entendida de diferentes formas ${ }^{5}$; las teóricas coinciden en que está permite, desde la perspectiva crítica social, evidenciar las desigualdades e inequidades que las mujeres históricamente han padecido frente a la participación de los hombres en la sociedad, en intersección con otras categorías de subordinación y de poder como la pertenencia étnicoracial, clase social, condiciones físicas, nacionalidad, entre otras.

De igual forma, la perspectiva de género permite identificar la construcción social, cultural e histórica de la identidad de género articulada a las vivencias y subjetividades de las personas (Kessler y McKenna, 1978).

Teniendo esto en cuenta, se entiende el género de acuerdo con los planteamientos de Scott (1986): "como un elemento constitutivo de las relaciones sociales basadas en las diferencias que distinguen los sexos y el género, es una forma primaria de relaciones significantes de poder" (p. 289), "que estructura la percepción y la organización, concreta y simbólica, de toda la vida social" (p. 293).

Sumado a lo anterior, se sostiene que en toda sociedad y cultura se estructura un orden de género que, según Buquet (2016), es“un sistema de organización social que subordina a las mujeres como colectivo frente al colectivo de los hombres y que construye diferencias arbitrarias cuyo resultado es el desempeño de papeles sociales diferenciados y jerarquizados que se reproducen en todos los ámbitos del ser y del quehacer humano. Esta diferenciación es producto y, a la vez, productora de las distinciones de género"(p. 28).

Buquet, a su vez, establece tres dimensiones que lo conforman: la simbólica, el imaginario colectivo y la dimensión subjetiva. En la simbólica

\footnotetext{
5 "El género ha sido analizado como un atributo de los individuos (Bem 1974, 1983), como una relación interpersonal (Spelman 1988), y como un modo de organización social Firestone 1970; Eisenstein (1979). El género ha sido definido en términos de estado (Lopata y Thorne 1978), los roles sexuales (Epstein .1971; Janeway 1971, Amundsen 1971), y los estereotipos sexuales (Friedan,1963; Anderson,1983). Ha sido concebido como una estructura de la conciencia (Rowbotham 1973), como psiquis triangulada (Chodorow, 1978), y como ideología internalizada (Barrett 1980; Beca 1993). Se ha discutido como un producto de la atribución (Kessler y McKenna, 1978), como socialización (Giltigan 1982; Ruddick 1980). El género ha sido descrito como un efecto del lenguaje (Spender, 1980; Daly 1978), una cuestión de la conformidad del comportamiento (Epstein 1971; Amundsen 1971), una característica estructural del trabajo, poder y la catexis (Connell 1987), un modo de percepción (Kessler y McKenna, 1978; Bem, 1993). Se ha caracterizado como diferencia (Irigaray, 1985a, 1985b) y como las relaciones de poder que se manifiestan en la dominación y lasubordinación (MacKinnon, 1987, Gordon 1988)" (Hawkesworth, 2006, p. 146).
} 
se funda la distinción de carácter dicotómico y jerarquizante entre los significados asociados con la pareja simbólica femenino/masculino; el imaginario colectivo -entendido como imágenes socialmente compartidas-, que se manifiesta de maneras específicas en los distintos colectivos humanos, pero que siempre produce prácticas sociales profundamente diferenciadas entre hombres y mujeres, organizadas y reforzadas a través de las instituciones; y la dimensión subjetiva como mecanismo de internalización de estas diferencias, cristalizadas en las identidades de género, que, con mayor o menor apego al modelo tradicional, participan activamente en la reproducción - y resistenciafrente a los mandatos del orden de género (Buquet, 2016, p. 29).

De acuerdo con lo planteado, para el análisis de los documentos se tuvo en cuenta aspectos como el enfoque o perspectiva de atención a la población joven, la conceptualización o cómo se entendían el género y la identidad de género y, finalmente, cómo se pretende llegar a la atención de la diferencia y a la equidad.

\section{El enfoque o perspectiva sobre la que se lleva a cabo la intervención}

Al analizar los documentos descritos, se encuentra que la perspectiva de atención predominante es la de derechos, dentro de la cual se menciona la igualdad, la inclusión, la no discriminación, el respeto por la diferencia y por las libertades fundamentales, y los derechos fundamentales como la educación, la protección y la formación integral. Esta perspectiva de derechos alude a que todas las personas menores de 18 años son sujetos de derechos, incluyendo aquellas comunidades, como las indígenas, que tienen su propia jurisdicción.

De esta forma, los documentos estudiados plantean, como aspecto central, la garantía y restitución de derechos, privilegiando los de niñas, niños, adolescentes y jóvenes, de la cual somos responsables la sociedad, el Estado y la familia, en el marco de la corresponsabilidad y la concurrencia de cualquier actor para exigir el cumplimiento de los derechos; sin embargo, la principal responsabilidad es la del Estado. Este enfoque de derechos contempla también el ejercicio de estos de forma responsable por parte de las personas menores de 18 años, con apoyo del ejercicio de formación pedagógica de la familia, el Estado y la sociedad. 
Es interesante encontrar que la mayoría de los textos atañen la garantía de derechos a la familia, así como la responsabilidad del joven infractor tanto de sus acciones contra la ley como de la vigilancia para la no reiteración de estas, de tal manera que se desconoce el principio fundamental dela Ley 1098 de 2006 que corresponde al Código de Infancia y Adolescencia ( Congreso de Colombia, 2006) y la Ley 1878 de 2018 que corresponde a la Ley de Infancia y Adolescencia (Congreso de Colombia, 2018) relacionado con la corresponsabilidad entre Estado, sociedad y familia.

Se halla igualmente que sobre la familia recae la asignación de roles de género, atribuyendo a esta las violencias relacionadas con la identidad y la orientación sexual. Es evidente que estos escritos desconocen la sociedad, la política, la religión, la cultura y la economía inmersas en el orden patriarcal y el sistema económico capitalista como las causas sobre las que se erigen las violencias no solo contra las mujeres, sino contra cualquier persona subalternizada por motivos de orientación sexual, pertenencia étnicoracial, identidad de género, edad, capacidad funcional, etcétera. Lo anterior significa que la familia no es la principal responsable de esta situación, pues está inmersa en un conglomerado de aspectos socioculturales que configuran la violencia estructural.

Lo anterior demuestra que el Estado y la sociedad delegan sus responsabilidades a la familia mediante la culpabilización y la impartición de algunas estrategias tenues de prevención; por ejemplo, el Lineamiento Modelo de Atención para Adolescentes y Jóvenes en Conflicto con la Ley-Srpa- menciona la intervención y trabajo hacia las comunidades en aras de la prevención mediante propuestas educativas, culturales, deportivas, de salud y de formación para el trabajo. Es importante tener en cuenta que este paradigma de la prevención libra de responsabilidad al Estado y a la sociedad sobre la vinculación de adolescentes y jóvenes en las actividades delictivas y delega la intervención en el Tercer sector.

Un aspecto más hallado en los documentos revisados es la terminología empleada para referirse a (las/os) adolescentes y (las/os) jóvenes hacia quienes se dirigen sus atenciones. Se identifican términos como "el adolescente", "sujetos", "individuos", "niña", "las y los adolescentes", "niños, niñas y adolescentes", "los adolescentes y los jóvenes", "las y los adolescentes y jóvenes". De los mencionados, este último es el que hace una alusión más amplia e incluyente; no obstante, solo se encuentra en el aparte inicial del lineamiento Modelo de 
Atención para Adolescentes y Jóvenes en Conflicto con la Ley-Srpa, específicamente donde se abordan temas de género e inclusión al principio del documento. Paradójicamente, en el resto de este se emplea la denominación "los adolescentes y jóvenes", predominando así la nominación masculina que pone en el centro de la atención y la intervención a un sujeto masculino y androcéntrico, desconociendo a las mujeres jóvenes y sus particularidades y diferencias como parte de la población foco de atención en el sistema de responsabilidad penal adolescente.

En este orden de ideas, es central plantear la equidad como paradigma ideal para la atención, entendido este como el reconocimiento, la justicia y la garantía de derechos a todas las personas por igual; para el caso, de las mujeres jóvenes de forma idéntica que a los hombres jóvenes. Por lo tanto, si bien la omisión o generalización de lo masculino sobre lo femenino no implica la no garantía de derechos, sí indica la invisibilización o minorización ("la niña") de lo femenino. De esta forma, los textos analizados emplean la terminología masculina para referirse a toda la población joven privada de la libertad; esta pretende generalizar lo femenino y lo masculino sobre la base de lo masculino como sujeto válido, posiblemente por representar el mayor porcentaje de cometimiento de delitos (86\%), en contraste con las mujeres que corresponden al 14\% (Instituto Colombiano de Bienestar Familiar, s.f., 2018), por lo tanto, genera mayor impacto en la seguridad ciudadana y, por ende, en la elaboración de las leyes. De esta forma, es posible afirmar que en los escritos se asume el lenguaje masculino como "incluyente", pero realmente es excluyente y generalizador, obviando así las particularidades de cada población.

En relación con lo anterior, se hace fundamental reconocer el lenguaje como la expresión del pensamiento, el reflejo de los usos y las costumbres de una sociedad,así como de las culturas. De igual forma, también es fuente de violencia simbólica, forma a través de la cual se ha naturalizado la invisibilización, discriminación y la desigualdad histórica de las mujeres, cuyosorígenesradican en los roles y estereotipos de género que limitan y encasillan a las personas a partir de sus diferencias anatómicas.

Otro aspecto relevante hallado es lo femenino expresado bajo el apelativo "la niña"6 haciendo referencia a mujeres jovenes entre los 14 y 18 años, aspecto que puede interpretarse

\footnotetext{
${ }^{6}$ La etapa de la niñez de acuerdo con la Organización Mundial de la Salud (OMS) va desde los 0 hasta los 10 años, seguida de la pubertad, la adolescencia, la juventud y la adultez. 
como el mensaje de ser pequeña, inmadura, manejable y vulnerable (como lo es aquella menor de 10 años); lo que evidenciala minorizaciónque repercute en el desconocimiento de la población de mujeres jóvenes de 18 años que se encuentran en el sistema.

A esto se suma que lo femenino es invisibilizado, especificándose solo en el apartado relacionado con género e inclusión hallándose solo en uno de los documentos analizados ${ }^{7}$. Esta es una evidencia más de la invisibilización de las mujeres, pues su carácter se enuncia en un solo documento cuando se hace referencia a la conceptualización de género e identidad. Es entonces que se pone en cuestión la inclusión de las mujeres inclusive en textos sustentados sobre la perspectiva de derechos.

Continuando con el análisis, se halla una única denominación específica de lo femenino en los apartes de los textos que abordan la atención a las madres gestantes y lactantes, reconocimiento que se enmarca en la asignación de los roles tradicionales de género. Es decir, la mujer solo se visibiliza en el rol de madre; no así en la educación, el trabajo o la participación social.

Esto demuestra la esencialización de la identidad femenina al interior de las instituciones sobre la base de la maternidad y el cuidado del bebé.Es decir, el papel en el que el Estado y sus instituciones visibiliza a las mujeres jóvenes se relaciona con la reproducción y el cuidado de sus hijos, para que, una vez crezcan, sean útiles al sistema y no repitan la historia de sus madres, especialmente aquellas circunstancias relacionadas con las rupturas del orden social.Con relación a lo anterior, sería interesante hallar planteamientos relacionados con las oportunidades que se incitan a brindar la sociedad y el Estado a las mujeres y madres jóvenes.

\section{La conceptualización de género}

La conceptualización de género se especifica en dos de los seis documentos analizados; estos son: el Código de Infancia y Adolescencia, Ley 1098 de 2006, y en el Lineamiento Modelo de Atención para Adolescentes y Jóvenes en Conflicto con la Ley-Srpa.

En el primero, se conceptualiza la perspectiva de género como: "el reconocimiento de las diferencias sociales, biológicas y psicológicas en las relaciones entre las personas según el sexo, la edad, la etnia y el rol que desempeña en la familia y en el grupo social" (Congreso de la

\footnotetext{
${ }^{7}$ En el lineamiento Modelo de Atención para Adolescentes y Jóvenes en Conflicto con la Ley-Srpa.
} 
República, 2006, Art. 12, p.5). Esta perspectiva, si bien incluye aspectos a partir de los cualesse refleja la perspectiva del género, toma distancia de las diferencias naturalizadas a partir del orden de género; de ser así, se entendería la perspectiva de género como el reconocimiento de la diferencia a partir de las particularidades anatómicas y estás como base de las construcciones sociales y culturalesconsideradas como naturales y, por lo tanto,aparentemente inmodificables.

En el segundo texto, se amplía el posicionamiento a líneas de pensamiento más contemporáneas, que se sustentan en documentos realizados por instituciones públicas como la Alcaldía de Bogotá en el año 2014, el Instituto Interamericano de Derechos Humanos y Cejil en 1997 y la Oficina Regional para América del Sur del Alto Comisionado de las Naciones Unidas para los Derechos Humanos ACNUDH. Estos plantean que

el género se expresa en el reconocimiento de la diversidad, en el abordaje desde los espacios en los que se atiende, quien lo hace, así como la propuesta de atención que se plantea diferencialmente a cada persona según orientación sexual e identidad de género y como lo expresa en su rol familiar y social". Así, "La identidad de género puede entenderse ampliamente como el sentimiento subjetivo íntimo de ser "varón" o "mujer", estructurado por procesos de identificación y proyección en interacción con la identidad sexual"; lo que se expresa en sus relaciones con otros y en manifestaciones psico-emocionales particulares" (Secretaría Distrital de Planeación, citada en Instituto Colombiano de Bienestar Familiar, 2016b, p.53),

el término «género» se refiere a las identidades, las funciones y los atributos construidos socialmente de mujer y hombre y al significado social y cultural que se atribuye a esas diferencias biológicas (Comité para la Eliminación de la Discriminación contra la Mujer, citado en Instituto Colombiano de Bienestar Familiar, 2016b; p.55). 
el concepto género es una forma de entender a los seres humanos, a partir de la comprensión de que es la sociedad la que se encarga de asignar a las personas características fijas y el rol a desempeñar dentro de ella en función de su sexo; y, por tanto, de haber colocado a algunos individuos en una posición de subordinación histórica respecto de lo masculino. Así, teniendo en cuenta que es una construcción social, artificial y voluntaria, es por ello que se puede y debe modificar, principalmente cuando dicha asignación afecte, discrimine e incluso subordine a algunos frente a otros (Instituto Interamericano de Derechos Humanos y Cejil, citado en Instituto Colombiano de Bienestar Familiar, 2016b; p.56),

La identidad de género es la vivencia individual del género, es decir como cada persona la experimenta profunda e individualmente. Esta podría corresponder o no con el sexo asignado al momento del nacimiento ya que hay una vivencia personal del cuerpo. Por ello, esa vivencia individual puede llevar a la persona a modificar su apariencia o la función corporal a través de procedimientos médicos, quirúrgicos o de otra índole, (siempre que la misma sea libremente escogida) y otras expresiones de género, incluyendo la forma de vestir, de hablar y los modales etcétera. Así existen varias identidades de género como: el ser travesti, transexual, transgénero o intersexual (Oficina Regional para América del Sur del Alto Comisionado de las Naciones Unidas para los Derechos Humanos ACNUDH, citada en Instituto Colombiano de Bienestar Familiar, 2016b; p.56).

Esta múltiple conceptualización en el documento muestra que, si bien menciona en un aparte amplio la perspectiva de género y la identidad como construcciones socioculturales, estas conceptualizaciones no transversalizan la fundamentación del mismo, puesto que el centro de enunciación continúa siendo los varones desde la forma como se mencionan las personas, hasta las acciones que se realizan en el marco del Sistema de Responsabilidad Penal Adolescente.

Es clave identificar que la perspectiva de género mencionada en los documentos está dirigida y fundamentada en una concepción bigenerista, tradicional y propia del orden patriarcal que sustenta la dicotomía femenino/masculino, mujer/hombre, pese a algunas menciones a la diversidad. Es importante tener en cuenta que una perspectiva diversa debe tomar distancia de las 
dicotomías y los sistemas binarios, bigeneristas; por lo tanto, pese a las menciones aisladas sobre identidades no hegemónicas, los documentos, por ser los que dictan las acciones interventivas e ideológicas con la población, continúan reproduciendo el orden social dicotómico hombre/mujer como lo "normal", lo "correcto" y las relaciones de subordinación y de poder como lo "esperado".

Otro de los apartes del documento expresa desde un lenguaje masculino:

el documento de la Dirección de Equidad y políticas poblacionales señala: "Existen las mujeres que sienten discriminación hacia ellas en la crianza, en mayores violencias sexuales, menos oportunidades de trabajo y estudio, viven más pobreza crónica, tienen más obligaciones en el hogar como oficios y cuidado de los niños, menos oportunidades para disfrutar la ciudad y menos libertades que los hombres. (...) las vulneraciones en los hombres con más limitaciones permanentes, más exigencias en el trabajo, más altos niveles de pobreza reciente y más niveles de violencias como robos y atracos (Secretaría Distrital de Planeación, citada en Instituto Colombiano de Bienestar Familiar, 2016b; p. 53-54).

Este aparte, inserto en uno de los lineamientos y tomado de un documento de la Secretaría Distrital de Planeación (2014; citada en Instituto Colombiano de Bienestar Familiar, 2016b), muestra la perspectiva androcéntrica del Estado en la que se minimiza la subordinación de la mujer a las quejas cotidianas, para luego resaltar la difícil y permanente situación de los hombres.

Al final del capítulo referido al género, se resalta la importancia de brindar las herramientas para el libre desarrollo de la personalidad en cuanto a identidad de género y orientación sexual; no obstante, se cierra el aparte con lo siguiente:

Las adolescentes mujeres y los adolescentes hombres deben poder desarrollar su proceso pedagógico en espacios dignos y con acciones acordes al contexto cultural, su rol social y familiar asumido, que las y los lleve a reflexionar y definir acciones claras articuladas en la convivencia y les permita construir nuevos modelos de feminidad y masculinidad (Instituto Colombiano de Bienestar Familia, 2016b, p. 60). 
Los anteriores enunciados ponen en evidencia nuevamente la perspectiva binaria del género en la que solo se hace referencia y se reconoce a las mujeres y los hombres, de tal forma que se desconocen otras identidades, otros géneros y se continúaubicando a la mujer en un lugar tradicional subordinado.

\section{La atención diferencial}

En cuanto a los hallazgos relacionados con la atención diferencial, el Código de Infancia y Adolescencia plantea la atención de acuerdo con la edad y el sexo. El Decreto 2083 de 2015 menciona la educación inclusiva de tal forma que las "prácticas pedagógicas y administrativas se adapten a las características, condiciones y capacidades de los adolescentes y jóvenes en conflicto con la ley penal" (Ministerio de Educación Nacional, 2015, p. 4), así como al respeto a la diversidad y a la inclusión.

El Modelo de Atención para Adolescentes y Jóvenes en Conflicto con la Ley-Srpa presenta procedimientos acordes a la atención diferencial, tales como la caracterización de cada joven promoviendo que la "acción sin daño evite la perpetuación de estereotipos, al contrario, favoreciendo la inclusión que potencie sus capacidades y las oportunidades que le brinda el entorno" (Instituto Colombiano de Bienestar Familia, 2016b, p. 41).

Por otra parte, menciona la consideración de la identidad de género para la ubicación de las y los jóvenes en las unidades de servicios de común acuerdo con ellas y ellos, y la atención personalizada acorde a sus condiciones familiares, sociales, expectativas, necesidades, etnia, identidad de género y orientación sexual.

La atención diferencial también se menciona en la minuta patrón o menú de alimentación, en el que se estipula que a las mujeres se sirve menor cantidad de carnes y cereales que a los varones. Si bien se considera que esta decisión se tomó bajo una amplia gama de estudios nutricionales que la respalden, se continúa cuestionando esta instrucción sobre la base de la equidad alimentaria y surgen preguntas como: ¿las jóvenes deben comer menos carnes y cereales para mantener su figura delgada, tal como lo establece el patriarcado/capitalismo, para ser más atractiva ante los varones?, ¿las mujeres jóvenes tienen menos apetito que los hombres? O ¿se propicia con esto la disminución de costos al sistema de forma que su manutención genere menos inversión? ¿en qué argumentos se sustenta esta diferenciación? 
Si bien la perspectiva diferencial es necesaria para ampliar la equidad y justicia en cuanto a la perspectiva de derechos, es imperativo y urgente asumir la perspectiva interseccional ${ }^{8}$ en la que, sobre la base de las múltiples condiciones de subordinación que pueden albergar los cuerpos, se intersectan condiciones que ubican a las personas en lugares de desventaja en las dinámicas sociales contemporáneas, de tal manera que se estrechan las posibilidades de obtener los mismos derechos y privilegios, lo cual genera situaciones de discriminación, deshumanización y violencia hacia quien las ostenta.

Finalmente, y en contradicción con lo mencionado por el documento sobre la atención diferencial, los formatos de evaluación especificados e incluidos en el anexo de este lineamiento no presentan ítems relacionados con la perspectiva diferencial género o identidad de género para la caracterización de la población, ni aquellos relacionados con la perspectiva interseccional, es decir, pertenencia étnicoracial, clase social, etcétera. Esto demuestra que la atención diferencial es un enunciado más en el lineamiento que no se traduce en acciones concretas, pues no se incluyen en sus formatos de caracterización ni de actuación, ninguna de las condiciones que ameritan atención diferencial e interseccional, por lo tanto, es difícil que esta sea tenida en cuenta por los operadores del Sistema de Responsabilidad Penal Adolescente.

En el Lineamiento de Servicios para Medidas y Sanciones del Proceso Judicial-Srpa se menciona la atención diferencial y formación a las madres gestantes y lactantes en habilidades propias para el cuidado de sus hijos que si bien, bajo algunas perspectivas se rescata la atención que merecen las madres jóvenes, bajo ópticas más amplias el lineamiento solo centra la atención (presenta un aparte específico) cuando la mujer joven es madre.Lo anterior descuida otras acciones centrales, formación o educación para el desarrollo de proyectos que pueden llevar a cabo dichas jóvenes, por ejemplo el ejercicio de liderazgos, el emprendimiento o el acceso a la educación superior.

\footnotetext{
8 "la interseccionalidad constituye la base del reconocimiento de las diferencias y las diferenciaciones para analizar las posiciones subjetivas de enunciación, comprender las formas de producción del poder, el privilegio y la marginalización de las personas a través de los arreglos identitarios interseccionados a partir de las múltiples categorías sociales subyacentes. Sin duda, es una convocatoria epistémica para traspasar los límites del pensar occidental binario, hegemónico o esencialista, sobre todo porque,al comprender el género en su expresión articulada e inseparable de otras categorías sociales, relaciona el poder como vía para tomar distancia de la visión impuesta que considera a las mujeres como un grupo homogéneo y devela las razones por las cuales muchas situaciones vividas por mujeres y hombres de todas las condiciones, quedan invisibles y opacadas” (Munévar,2013, p.15).
} 
En cuanto a la atención equitativa, el Decreto 2383 de 2015 menciona la atención en iguales condiciones sin ningún tipo de discriminación; el lineamiento Modelo de Atención aborda la facilitación de la igualdad y la no discriminación como un trabajo conjunto entre "los jóvenes" y profesionales que trabajan con ellas/ellos. Esto indica que la equidad no solo es un componente que debe trabajarse como tema de talleres, también debe ser construido el producto de las coconstrucción con las y los profesionales de los Centros de Atención Especializados, esto para reafirmar la participación de las subjetividades de las y los interventores en el trabajo de las y los jóvenes.

De igual forma, se plantea la importancia del uso de un lenguaje incluyente en las intervenciones, lo que refuerza que la inclusión y la equidad van más allá de ser trabajos en papel; deben ser prácticas cotidianas que parten de las acciones tanto de las y los interventores como de las y los jóvenes privadas/os de la libertad.

Una de las acciones concretas que muestran la equidad es la promoción del ejercicio responsable de la sexualidad, para lo cual se propone el abordaje de tópicos incluyentes a las diversidades y herramientas de construcción de la libre personalidad; sin embargo, esto solo queda en manos de psicología y o de quien hace las veces de auxiliar de enfermería. De esta forma, las y los otros interventores y profesionales son marginados de estos procesos, lo que genera el desconocimiento del equipo ampliado sobre los temas, abordajes y formas de atención que aquellos profesionales han desarrollado con las y los jóvenes. Bajo esta dinámica, se arriesgan los avancesy construcciones alcanzadas, pues lasy los demás interventoras/es, al desconocer estos aspectos, pueden no tener en cuenta en sus prácticas cotidianas estos temas o generar contradicciones con los trabajos y discursos realizados por las/os interventoras/es a cargo de estos. Es así, como en ningún apartado se menciona las relaciones de poder y subordinación que se construyen en las relaciones de género, dejando de lado las brechas, las desigualdades y los sesgos culturales que se construyen a partir de la pertenencia a un género.

\section{El interior de los Centro de Atención Especializados. Lo pre escrito-lo real}

Es importante entender que, en Colombia, las instituciones en las que se lleva a cabo y que acompañan la sanción privativa de la libertad se denominan Centros de Atención Especializados (CAE), que, como instituciones totales, pretenden "proteger la comunidad contra quienes constituyen un peligro para ella" (Goffman, 2001, p.18). Así, las instituciones en las que las 
jóvenes son privadas de la libertad se pueden entender como "lugares de residencia y trabajo, donde un gran número de individuos en igual situación, aislados de la sociedad, por un periodo apreciable de tiempo, comparten en su encierro una rutina diaria, administrada formalmente" (Goffman, 2001, p. 13).

Estas instituciones totales desculturizan, higienizan, proporcionan un mundo propio, aíslan del exterior, imponen roles y rutinas ajenas, moldean, clasifican, despojan a las personas de sus propiedades y marcas que les significan sus identidades personales, conduciendo así a la pérdida de la autodeterminación, la razón y el lenguaje (Goffman, 2001). Estas características son cercanas a la vida cotidiana de las mujeres durante el tiempo en que se encuentran privadas de la libertad en las instituciones.

En el mismo sentido, Turner (2008) explica los modelos de la máquina correctiva cuyo fin es restaurar y reintegrar las personas a la sociedad mediante la higienización y estandarización de la fachada. Para el caso de las jóvenes en conflicto con la ley, esto implica la posibilidad del desprendimiento de sus hábitos y rutinas de autocuidado, además de la uniformidad en la vestimenta, que propicia una mudanza circunstancial y temporal en los hábitos (Suárez, 2016). Entonces, los Centros de Atención Especializados, además de aislar a las jóvenes de sus contextos, pretende transformarlas ${ }^{9}$, logrando tal vez, cambios en su apariencia y probablemente en algunos comportamientos, siendo posible el olvido de estos una vez retornan a sus contextos.

Las instituciones tienen programas que designan "un tipo particular de socialización, una forma específica de trabajo sobre los otros" (Dubet, 2006, p. 32). Si bien estos programas se sustentan en unos principios y valores que en el caso colombiano están determinados por el Estado y acuñados por la misma institución, de forma dinámica se configuran y reconfiguran por las personas pertenecientes a estas. Para el caso de los Centros de Atención Especializados (CAE), son las y los jóvenes privados de la libertad y las/los agentes interventores, todos con una serie de valores, principios y patrones culturales que los transversalizan y se extienden a sus lugares en el proceso de intervención, entonces "el trabajo real borra el trabajo prescrito" (Dubet, 2006, p. 20).

\footnotetext{
${ }^{9}$ En este momento es relevante precisar la diferencia entre cambioy transformación; para esto se seguirán los planteamientos de Edgar Morin (2001) en su libro El Método. La naturaleza de la naturaleza, en el que plantea el cambio como transitorio, circunstancial y sectorizado; y la transformación como amplia, sistémica y perdurable. 
Entonces, al pensar en una "institución total" (Goffman, 2001, p.13) y sus acciones de higienización y estandarización de la fachada a las que se refiere Turner (2008) en sus planteamientos acerca de los modelos de la máquina correctiva, se halla un punto de encuentro al pretender alcanzar el mismo fin: restaurar y reintegrar las personas a la sociedad. A partir de este planteamiento, se entiende que, para el caso de las jóvenes en conflicto con la ley, esto implica el desprendimiento de sus hábitos y rutinas de autocuidado, además de la imposición de una vestimenta uniforme para todas, que acarrea una mudanza en los hábitos que, más que un cambio estructural, representa simplemente un cambio de semblante (Suárez, 2016).

Acker (1990) sostiene que las instituciones y las personas que integran las organizaciones reproducen el género en sus intervenciones, es decir, transmiten sus ideales de lo femenino y lo masculino en actividades concretas, creencias y pensamientos mediante las interacciones, el lenguaje y los símbolos, lo que lleva a reforzar estas estructuras generizadas y las relaciones de poder y subordinación basadas en el género. Estas se sustentan, además, en los valores, la historia, la cultura y las prácticas tanto de las y los interventores como de las organizaciones a las que pertenecen.

Las instituciones totales que acompañan los procesos de las jóvenes, a partir de políticas definidas e interventoras/es en cuya identidad de género sustentan sus acciones, acuñan a partir de los lineamientos analizados un orden ${ }^{10}$ de género que, de acuerdo conReeves (1981), corresponde a un modelo para la organización de las expectativas de los roles sexuales, en el que inscriben sus intervenciones y por ende el rol de género esperado de las jóvenes en conflicto con la ley. Lo anterior hace presumir que la institucionalización total y las acciones que se desarrollan al interior de estas son generizadas y permiten mantener el guion de género instaurado socialmente.

De esta forma,transmiten el orden de género, un orden de estatus (Segato, 2003), una “estructura de relaciones entre posiciones jerárquicamente ordenadas” (p.14) en la que el lugar superior lo ostenta el patriarca, que, desde lo simbólico, reproduce el orden, la norma y la autorreproducción de dicho lugar, de una simbología de poder.

Estos están permeados y naturalizados por la dimensión simbólica sobre la base de lo dicotómico, el imaginario colectivo, en el que se acuñan unas formas de ser mujer y de ser

\footnotetext{
${ }^{10}$ Peggy Reeves en su escrito lo denomina textualmente guion de género. 
hombre compartidas socialmente, se introyecta en la identidad de género de cada uno de sus miembros (Buquet, 2016).

Por lo tanto, al ser estas instituciones las delegadas del Estado para devolver y mantener el orden social $^{11}$, propenden por la reproducción de este que, de acuerdo con sus dinámicas internas, puede entrar en tensión con loslineamientos que preceden sus acciones interventivas, lo que remite a lo planteado por Dubet (2006) al mencionar que "el trabajo real borra el trabajo prescrito" (p. 20).

La comprensión del proceso de intervención social se puede ampliar con base en los planteamientos de Foucault (2003), quien a lo largo de su estudio genealógico de la prisión y el castigo plantea que, si bien este último dejó de ser físico, continúa recayendo sobre los cuerpos, siendo entonces los instrumentos de este, no solo al privarlos de la libertad, instigarlos a las acciones con alta demanda física o al separarlos de sus contextos, sino al pretender, entre otros aspectos, la transformación de su apariencia y semblante.

Otro de los aspectos que se pretende 'transformar', además de los mencionados, son las acciones de las jóvenes que constituyen su vida cotidiana, sus formas de actuar y accionar, que se convierten entonces en instrumentos para la reproducción del orden social y de género a través de los que ellas deben cumplir con sus roles de cuidadoras, productoras y reproductoras. Esto pretende la docilidad y sumisión ante las exigencias de un gran grupo de interventoras/es, representantes del orden social estatal (y de género).

De esta forma, el Sistema de Responsabilidad Penal Adolescente es una fiel representación delsistema social pues, más allá de castigar una acción indebida a los ojos de la sociedad y el Estado, pretende actuar sobre la joven, controlarla, neutralizarla y modificarla, cambiando con estas pretensiones su estatus de infractora a la de delincuente.Es así como más allá de la sanción por una acción, se desea cambiar un sujeto en su esencia y constitución, es decir, transformarla e introducirla entonces al sistema capitalista/patriarcal, instigándola a la productividad económica, al cuidado del otro y a la reproducción de la mano de obra, siendo entonces dicha dinámica aquella que mantiene las instituciones tanto estatales como aquellas que pertenecen al Tercer sector.

\footnotetext{
${ }^{11}$ De acuerdo con Guzmán y Rodríguez (2014), se entiende como "el problema de la reproducción de la sociedad con consecuencias normativas para esta, sus instituciones y actores" (p. 157). 
Este proceso de intervención correctiva es sometido permanentemente al examen como “instrumento simple del poder disciplinario" (Foucault, 2003, p.157), es decir, el Estado vigilia el estricto cumplimiento de este. Así, como herramienta verificadora de la disciplina, es esta la forma de corroborar que todo el aparataje institucional ha surtido efecto. No obstante, este tiene un doble filo: objetivamente pretende mostrar el cambio o aprendizaje de las jóvenes, pero no garantiza el ejercicio de este cambio o la transformación una vez egresan de las instituciones. En este punto es necesario tener en cuenta los aspectos que entran en juego cuando una joven egresa de los centros de atención especializados; si bien la institución brinda las herramientas necesarias para la participación positiva en la sociedad, la sociedad colombiana - transversalizada por la dimensión simbólica y el imaginario colectivo, que no solo hace referencia al orden de género, sino también a lo que simboliza el cometimiento de un delito y la consecuente privación de la libertad- reduce las posibilidades de participación, ya escasas para las mujeres jóvenes que no han estado en el sistema de responsabilidad penal adolescente. Así, sin oportunidades laborales o de formación, el proceso de intervención plasmado en los lineamientos pierde el sentido original de restauración y pasa a tener un sentido exclusivamente punitivo, perpetuando y reproduciendo las brechas, desigualdades y relaciones de poder y subordinación.

Foucault (2003) menciona la participación de los "criminales" en el castigo que se ejerce sobre estos al pertenecer al cuerpo social; en este punto, ponemos en discusión desde la postura feminista dicho planteamiento ¿Son las mujeres jóvenes, pobres, racializadas, con poca formación escolar, escasa experiencia laboral, con diversidad sexual, con responsabilidad penal, causa y efecto de la "cuestión social", partícipes del cuerpo social?

Si ellas formaran parte del cuerpo social, serían reconocidas como tal, lo que se evidenciaría en la participación de su castigo, en las dinámicas diferenciales al interior de las instituciones, es decir, no tendrían fines de uniformidad ni de control del cuerpo femenino en aras del entrenamiento en las labores de producción en la esfera privada y reproducción del cuerpo de trabajadores. Si participara, el castigo sería emancipador y empoderador, en la medida en que la potencializaría en el cuerpo social al que idealmente pertenece.

Entonces, se hace necesario realizar propuestas seguidas de acciones que conduzcan a la equidad y reconocimiento de las mujeres como parte importante del cuerpo social, no solo para las labores de cuidado y la reproducción de la mano de obra. 


\section{Reflexiones incidentes para política pública}

En este sentido es fundamental que los lineamientos planteados en las políticas de atención e intervención con jóvenes infractoras de la ley, realmente se transversalicen con perspectiva de género, la cual muestra cómo y de qué forma cada fenómeno específico está atravesado por las relaciones de poder, subordinación y desigualdades entre los géneros, que caracterizan los sistemas patriarcales (Serret, 2011). Así mismo, visibiliza a las mujeres, sus actividades, sus vidas, sus espacios y la forma en la que contribuyen a la creación de realidad social. De esta forma, es necesario trascender los enunciados formales y pasar a acciones concretas en los procesos de intervención con las mujeres, de tal manera que los procesos realizados con ellas, especialmente los educativos y de inserción en la sociedad permitan trabajar en cómo reducir las brechas de género tan significativas en Colombia ${ }^{12}$, las cuales se expresan en el acceso a empleo, a salud ya la participación política, todos estos aspectos ausentes en los lineamientos establecidos por el Estado para la intervención.

Entonces, si se quisiera trascender lo mencionado hacia la construcción de políticas públicas, se hace necesario dar inicio a la reflexión recordando la conceptualización de política pública que realiza Parsons (2007); él hace referencia a esta como un instrumento para el abordaje de las problemáticas y la toma de decisiones relacionadas con los recursos cercanos en el escenario de dicha problemática.

Así, se requeriría entonces iniciar por el reconocimiento de la problemática que, tal como se ha enunciado a lo largo de este escrito, trasciende la infracción de las jóvenes y llega hasta la invisibilización de sus particularidades más allá del "deber ser" acuñado por el pensamiento patriarcal. Es así como se hace necesario iniciar por la realización de un diágnostico que lleve a la delimitación de los problemas concretos que se deben atender en las mujeres jóvenes infractoras de la ley, quelos responsables de su definición, diseño, implementación y seguimiento asuman el

\footnotetext{
${ }^{12} \mathrm{Si}$ bien las brechas de género en el mundo han disminuido, en Colombia siguen aumentando, de acuerdo con el índice global de brechas de género 2018, nuestro país se situó en con un 72,9\% en el puesto 40 constituyendo importantes desafíos para disminuirlas; entre estos, la participación política de las mujeres sigue siendo muy baja con relación a otros países de América Latina. En el 2015, de acuerdo con datos de ONU Mujeres (2015),tan solo se obtuvieron el $14 \%$ de concejalas, el 17\% de diputadas, el 10\% de alcaldesas y el $9 \%$ de gobernadoras. Cifras aún muy por debajo de la paridad que haría justicia a la proporción de mujeres dentro de un ámbito fundamental en términos de decisiones para la sociedad colombiana. Con relación a los derechos económicos, el acceso de las mujeres al empleo formal y su participación en el Mercado laboral, si bien ha ido creciendo, es aún limitada.
} 
compromiso de superar las desigualdades de género y tomar en cuenta de manera sistemática los efectos diferenciados que ejercen las acciones de cada sector en mujeres y hombres. Para que así la actuación rompa con las perspectivas generalizantes, descontextualizadas y creadas a partir de personas consideradas y asumidas como homogeneas.

A partir del reconocimiento, de problemas concretos y específicos de las jóvenes infractoras se propone pensar en la construcción de políticas (o al menos lineamientos) cuyos enfoques se aproximen a las diferencias y al reconocimiento de la subordinación histórica de todos aquellos sujetos diferentes al masculino, blanco, heterosexual y racional. Es decir, políticas que reconozcan las mujeres tanto sujetas de derechos, como con particularidades étnicoraciales, de orientación sexual, de capacidad funcional, de clase socioeconómica y de ciclo de vida entre otras. Así las cosas, se propone que las políticas reconozcan las necesidades de las mujeres jóvenes sobre la base de sus capacidades, talentos y potencialidades en su contexto; trascendiendo los supuestos hegemónicos del rol de la mujer joven en la sociedad y permitiendo ser, y si se quiere, proyectarse desde una perspectiva emancipatoria hacia la reivindicación del ser mujer en la sociedad.

De esta manera, las políticas de intervención con jóvenes infractoras de la ley deben incluir en todos sus aspectos: marco de referencia, justificación, objetivos, metas, medidas de políticas y programas la perspectiva de género, de tal manera que permita tanto en la fundamentación como en la actuación,reconocer a personas diferenciadas por identidad de género, pertenencia étnico racial, clase social, entre otras, para así visibilizar a las mujeres jovenes infractoras en sus vidas, sus espacios, sus realidades de subordinación, pero también cómo ellas desde sus contextos pueden contribuir a la creación de realidades sociales; para así proponer acciones que permitan cerrar las brechas, las inequidades que han estado presentes en sus cortas vidas y que han tenido impacto en las infracciones contra la ley en las que han incurrido.

\section{Conclusiones}

El análisis de los documentos que brindan los lineamientos para la atención de las y los jóvenes, si bien incluyen en apartes específicos (escasos y limitados en comparación con la extensión del documento) aspectos relacionados con el género y la atención diferencial, no transversalizan la 
perspectiva o el espíritu del documento, es decir, en el resto del texto se hace referencia exclusiva a los hombres jóvenes.

Así mismo, en los pocos apartados en los que se evidencian elementos relacionados con lo mencionado, estos solo se ciñen a la maternidad, la responsabilidad en el cuidado de los hijos, y las porciones menores de proteína y cereales que deben consumir las mujeres. De esta forma, pese a lo enunciado, es posible afirmar que se continúa reproduciendo el orden de género bigenerista, jerárquico y subalternizante, que influye en el desconocimiento de la importancia de tener en la mira los aspectos que transversalizan, se intersectan y disminuyen las condiciones de equidad de las jóvenes en el sistema de responsabilidad penal adolescente.

De igual forma, se evidencia que los planteamientos de las leyes y lineamientos en cuanto a perspectiva de género, si bien textualmente es mencionada en un aparte reducido del texto, no refleja la comprensión del género más allá de la diversidad. La mención se realiza como un aspecto al que 'hay que dar trámite', es decir, se presenta como una mención obligada, mas no representa la esencia o espíritu del lineamiento. Por lo tanto, la perspectiva diferencial se consolida en los documentos en breves menciones que luego se diluyen en el lenguaje genérico.

Las menciones en los lineamientos de apoyo y atención solo referencian lo femenino en aspectos que históricamente han concernido a las mujeres: el cuidado de los hijos y la ingesta de menos alimentos que los hombres. Descuidando la formación de mujeres líderes, y las diferentes condiciones que además del género transversalizan las mujeres jóvenes, por ejemplo: la pertenencia étnicoracial, la clase social, las capacidades funcionales, etcétera.

Las formas como llegan los lineamientos a las jóvenes que han infringido la ley están transversalizadas por numerosos factores, que además de estar consignados en los textos reglamentarios, se refuerzan con las características propias de cada institución, en cómo estas moldean a sus miembros y están articuladas a las subjetividades de cada interventora. Todo lo anterior sustentado en las trayectorias y los contextos socioculturales e históricos particulares que pueden alterar sus principios fundacionales.

Un aspecto fundamental que se suma a la conceptualización de la política pública la realiza García (2008) quien menciona que estas dan respuesta a problemas de interés colectivo e implican procesos participativos; no obstante, si se quisiera tener en cuenta estos planteamientos, 
se precisaría comprender las problemáticas que transversalizan las mujeres jóvenes como menores o de poca monta, desconociendo que el $51 \%$ de la población colombiana está compuesta por mujeres, que serán, son o han sido jóvenes; y que han conocido de cerca las problemáticas como la discriminación, invalidación, desconocimiento y falta de reconocimiento.

De igual forma, al plantearse el proceso de construcción como participativo, se hace necesaria la participación de las mujeres jóvenes con múltiples interseccionalidades en la construcción de la política; no obstante, se considera su opinión como no válida, pues son "jóvenes sin experiencia", invalidando su existencia y considerando que está nublada su perspectiva "objetiva" por las múltiples subalternidades que habitan en ellas. Entonces, fallidamente se regresa a las políticas planteadas por sujetos, que si bien poseen "buena voluntad", no ha pasado por sus cuerpos y vida cotidiana la experiencia de ser una mujer joven privada de la libertad con múltiples condiciones que la hacen vulnerable y subalternizada.

Así las cosas, se recomienda la formulación de políticas con una real participación de la población foco de esta, que para el caso son las mujeres jóvenes privadas de la libertad. Es decir,

que se formulen acciones que den respuesta directa y contundente a sus problemáticas actuales, y permitan su participaciónactiva en la sociedad a partir de sus capacidades, talentos, posibilidades, expectativas y necesidades, de tal manera que se permita el fortalecimiento y consolidación de su capacidad de agencia. Es este sentido, la atención e intervención a las mujeres infractoras debe permitir acceso a la educación, a la salud a la seguridad social y ciudadana, incidiendo así en las condiciones sociales que generan desigualdad y precarización, aspectos que contribuyen a perpetuar la reincidencia en la infracción de la ley.

\section{Referenciasbibliográficas}

Acker, Joan. (1990). Hierarchies, Jobs, bodies: A Theory of Gendered Organizations. Gender \& society, 4(2), 139-158.

Buquet, Ana. (2016). El orden de género en la educación superior: una aproximación interdisciplinaria. Nómadas, (44), 27-43.

Congreso de La República de Colombia. (2006). Ley 1098. http://www.alcaldiabogota.gov.co/sisjur/normas/Norma1.jsp?i=22106 
Congreso de la República de Colombia. (2012). Ley 1577. http://www.secretariasenado.gov.co/senado/basedoc/ley_1577_2012.html

Congreso de la República de Colombia. (2018). Ley 1878. https://www.icbf.gov.co/cargues/avance/docs/ley_1878_2018.htm

De Sousa Santos, Boaventura. (2006). Conocer desde el Sur. Para una cultura política emancipatoria. Perú: Fondo Editorial de la Facultad de Ciencias Sociales,UNMSM, Programa de Estudios sobre Democracia y Transformación Global.

Departamento Administrativo Nacional de Estadística -DANE-.(2018). Censo Nacional de Población y Vivienda.

Dubet, François. (2006). El declive de la institución: profesiones, sujetos e individuos en la modernidad. España: Editorial Gedisa, SA.

Foucault, Michel. (2003). Vigilar y castigar: nacimiento de la prisión. México: Siglo XXI(original publicado en 1990).

García, Evangelina. (2008).Políticas de Igualdad, Igualdad y GenderMainstreaming. ¿De qué estamos hablando? Marco Conceptual.Edición Revisada y actualizada. Agencia Catalana de Cooperación- Programa de las Naciones Unidas para el desarrollo y Fondo España PNUD.

Goffman, Erving. (2001). Internados: ensayos sobre la situación social de los enfermos mentales. Barcelona: Amorrortu.

Guzmán, Álvaro y Rodríguez, Alba. (2014). Reconfiguración de los órdenes locales y conflicto armado: el caso de tres municipios del Norte del Cauca (1990-2010). Sociedad y Economía, (26), 155-184.

Hawkesworth, Mary. (2006). Feminist inquiry: From political conviction to methodologicalinnovation. Estados Unidos: Rutgers UniversityPress.

Instituto Colombiano de Bienestar Familia.(2016a). Lineamiento de Servicios para Medidas y Sanciones del juceso SRPA. https://www.icbf.gov.co/sites/default/files/procesos/lm14.p_lineamiento_para_servicios_ medidas_y_sanciones_proceso_judicial_srpa_v1.pdf 
Instituto Colombiano de Bienestar Familiar. (2016b). Lineamiento Modelo de Atención para Adolescentes y Jóvenes en Conflicto con la Ley-SRPA. https://www.icbf.gov.co/sites/default/files/procesos/lm15.p_lineamiento_modelo_atencio n_adolescente_y_jovenes_srpa_v2_0.pdf

Instituto Colombiano de Bienestar Familiar. (2018).Tablero SRPA-Sistema de Responsabilidad Penal para Adolescentes. https://www.icbf.gov.co/bienestar/observatorio-bienestarninez/tablero-srpa

Kessler, Suzanne y McKenna, Wendy. (1978). Gender: An Ethnomethodological Approach. Estados Unidos:University of Chicago Press.

Ministerio de Educación Nacional. (2015). Decreto Número 2383. http://wp.presidencia.gov.co/sitios/normativa/decretos/2015/Decretos2015/DECRETO\%2 02383\%20DEL\%2011\%20DE\%20DICIEMBRE\%20DE\%202015.pdf

Morin, Edgar. (2001). El Método. La naturaleza de la naturaleza. Madrid: Ediciones Cátedra, sexta edición.

Munévar, Dora. (2013). Interseccionalidad y otras nociones. En La interseccionalidad en debate. Actas del Congreso Internacional "Indicadores Interseccionales y Medidas de Inclusión Social en Instituciones de Educación Superior” (pp. 56-65), editado por Marta Zapata; Sabina García; Jennifer Chan. Berlín: Freie Universität Berlin. http://www.miseal.org/index.php/publicaciones/36- documentacion/publicaciones/147

ONU Mujeres.(2015). Informe anual 2014-2015. https://www2.unwomen.org//media/annual\%20report/attachments/sections/library/un-women-annual-report-20142015-es.pdf?vs=5603

Organización de Naciones Unidas. (2018)Recuperado en: https://population.un.org/wpp/Parsons, W. (2007). Una introducción a la teoría y la práctica del análisis de políticas públicas. México: Flacso.

Reeves, Peggy. (1981). Female Power and Male Dominance: On the Origins of Sexual Inequality. United Kingdom: University of Cambridge Press. 
Scott, Joan. (1986). El género: Una categoría útil para el análisis histórico. En: Lamas Marta (Comp.). El género: la construcción cultural de la diferencia sexual.México: PUEG, pp. 265-302.

Serret, Estela. (2011). Hacia una redefinición de las identidades de género. GénEros, 9, 71-97.

Segato, Rita. (2003). Las estructuras elementales de la violencia: ensayos sobre género entre la antropología, el psicoanálisis y los derechos humanos. Argentina: Universidad Nacional de Quilmes.

Suárez, Carlos. (2016). Estigma, communitas y modos de corrección para los habitantes de la calle en Bogotá (2000-2010). Sociedad y Economía, (32), 195-216.

Turner, Victor. (2008). Dramas, campos e metáforas: ação simbólica nasociedade humana. Brasil: EdUFF.

Weber, Max. (2014). Economía y sociedad. México: Fondo de Cultura Económica. 